Rechtsmedizin 2021 · 31:42-49

https://doi.org/10.1007/s00194-020-00434-9

Online publiziert: 17 . November 2020

(c) Der/die Autor(en) 2020

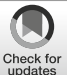

\section{F. Holz' $\cdot$ M. A. Verhoff' $\cdot$ J. Deschins ${ }^{1} \cdot$ G. Duttge ${ }^{2} \cdot$ M. Parzeller ${ }^{1}$}

' Institut für Rechtsmedizin des Universitätsklinikums, Goethe-Universität Frankfurt am Main, Frankfurt am Main, Deutschland

${ }^{2}$ Institut für Kriminalwissenschaften, Abteilung für strafrechtliches Medizin- und Biorecht, Georg-AugustUniversität, Göttingen, Deutschland

\title{
Der Doppelsuizid - Teil 2: rechtsmedizinische und kriminalistische Aspekte
}

\section{Einleitung}

„Weil wir so lange in Eintracht miteinander gelebt haben, o so lasset uns beide in einer Stunde dahinsterben; dann schau ich niemals das Grab des lieben Weibes, noch muß mich jene bestatten" [48]. Laut einer griechischen Sage war dies die gemeinsame Antwort des greisen Paares Philemon und Baucis auf die Frage des Göttervaters Zeus, welchen Wunsch er ihnen erfüllen dürfe. Und als ihre Zeit gekommen war, ,ward [er] zur Eiche, sie zur Linde, und noch im Tode stehen sie traulich zusammen, wie sie im Leben unzertrennlich waren" [48].

Das Versterben zweier Eheleute aus jeweils krankhafter innerer Ursache in einem kurzen zeitlichen Abstand wird in Anlehnung an oben genannte Sage als Philemon-und-Baucis-Tod bzw. -Phänomen beschrieben $[9,32,53]$. Wenn von 2 Personen eine natürliche Todesart willentlich auf einen früheren Zeitpunkt durch einen gemeinsamen und im Einverständnis begangenen Suizid vorverlegt wird, spricht man von einem Doppelsuizid oder gemeinschaftlichen Suizid [56]. Er ist von den teils uneinheitlich definierten und angewandten Begriffen des „erweiterten Suizids“ bzw. Homizid-Suizids (engl. homicide-suicide) abzugrenzen, bei dem die Tötung zumindest einer anderen Person ohne dessen Einwilligung erfolgt $[6,49,56]$.

Insbesondere wenn ein Beteiligter den Doppelsuizid überlebt, ist eine umfassende einzelfallbezogene juristische Würdigung erforderlich (ausführlich:
Teil 1 [12]), wobei die kriminalistische Aufarbeitung und die rechtsmedizinische Sektionsbegutachtung zur rechtlichen Entscheidungsfindung maßgeblich beitragen können. Dieser Teil beleuchtet anhand der im Institut für Rechtsmedizin Frankfurt am Main in dem Zeitraum von 25 Jahren (1995-2019) ausgewerteten Sektionsdaten rechtsmedizinische und kriminalistische Aspekte von Doppelsuiziden.

\section{Material und Methoden}

Das Institut für Rechtsmedizin Frankfurt am Main versorgt den kompletten südhessischen Raum sowie den südlichen Anteil von Mittelhessen. In dieser retrospektiven Studie wurden die Datenbank Forensik und die digitale Sammlung aller Obduktionsprotokolle des Instituts für die Jahre 1995-2019 nach den Schlagwörtern „Suizid“ („Doppelsuizid“, „erweiterter Suizid“, „Homizid-Suizid“) und „Tötung" durchsucht und, soweit erhältlich, die entsprechenden Obduktionsakten und polizeilichen Ermittlungsunterlagen analysiert. Einschlusskriterium war der deutliche Hinweis auf einen einvernehmlich vollendeten Doppelsui- zid in dokumentierter Form (z. B. Testament, Abschiedsbrief) oder einer vorangegangenen Ankündigung, unabhängig von den konkreten Umständen der Tatausführung. Eingeschlossen wurden zudem alle Fälle, bei denen eine der beteiligten Personen überlebte. Ausgeschlossen wurden Fallkonstellationen des erweiterten Suizids, in denen definitionsgemäß gegen den Willen des oder der Opfer gehandelt wurde.

Wenn nachfolgend von Fällen gesprochen wird, beziehen sich die Angaben jeweils auf die beiden beteiligten Doppelsuizidenten.

\section{Ergebnisse}

In dem ausgewerteten 25-jährigen Zeitintervall wurden ca. 16.400 Obduktionen durchgeführt. In die Studie konnten 23 Doppelsuizidfälle eingeschlossen werden, wobei zwischen 16 ,vollendeten Doppelsuizidfällen“ mit je 2 Verstorbenen und 7 „versuchten Doppelsuizidfällen" mit je einem Verstorbenen und einem Überlebenden zu differenzieren war. Dies entspricht einem Anteil von 0,24\% an allen Sektionsfällen. Ausgeschlossen wurden 49 Fälle eines vollendeten und

\section{Tab. 1 Geschlecht und Alter der verstorbenen Doppelsuizidenten $(n=39)$}

\begin{tabular}{l|llll} 
& \multicolumn{1}{c}{} & & + \\
\hline Anzahl & 22 & & 17 & \\
\hline Sterbealter (Jahre) & Altersrange & $18-86$ & Altersrange & $21-84$ \\
& Mittelwert & 57,8 & Mittelwert & 63,4 \\
& Median & 64,5 & Median & 65,0 \\
\hline Geschlechterverhältnis & $1,2: 1$ & & &
\end{tabular}


- Krankheit - eine der beiden beteiligten Personen erkrankt

- Krankheit - beide beteiligten Personen erkrankt

- Kombination familiäre, finanzielle und gesundheitliche Gründe

" finanzieller Ruin / Lebensumstände

Abb. 1 ॥ Übersicht der Motive für den Doppelsuizid

15 Fälle eines versuchten Homizid-Suizids [49]. In 3 Fällen war eine Differenzierung zwischen einem Doppelsuizid und einem Homizid-Suizid aufgrund der Vorgeschichte und der Auffindeumstände nicht sicher möglich, sodass diese ebenfalls nicht berücksichtigt wurden.

\section{Geschlecht, Alter, Beziehung}

In die 16 vollendeten und 7 versuchten Doppelsuizidfälle waren jeweils 2 Personen, somit insgesamt 46 Personen involviert. Geschlecht und Alter der Verstorbenen sind in - Tab. 1 angeführt. Das Alter der jeweiligen Suizidentenpaare unterschied sich in 19 Fällen um weniger als 5 , in den verbliebenen 4 Fällen um weniger als 10 Jahre.

Bei 19 Doppelsuiziden (82,6\%) lag eine Mann-Frau- (18 Ehepaare, 1 Liebespaar), bei $3(13,1 \%)$ eine MannMann- und bei einem (4,3\%) eine FrauFrau-Konstellation (eingetragene Lebenspartnerschaft) vor. Zur Dauer der Partnerschaften entließ sich den Akten nur entnehmen, dass 2 Ehen über mehr als 60 Jahre, 2 weitere über mehr als 50 Jahre bestanden.

Bei 3 Doppelsuiziden hatten sich die Beteiligten in einer psychiatrischen Einrichtung kennengelernt, die Beteiligten eines weiteren Doppelsuizids in einem Suizidinternetforum.

\section{Suizidankündigung}

In 17 Fällen $(73,9 \%)$ wurde ein von beiden Suizidenten unterschriebener, gemeinsamer Abschiedsbrief hinterlassen, wobei in 3 Fällen zusätzlich ein Testament hinterlegt war. In 5 Fällen $(21,7 \%)$ wurde der Suizidentschluss durch beide Suizidenten telefonisch bzw. über Textnachrichten gegenüber der Familie mitgeteilt. Ein Doppelsuizid von 2 Männern wurde zuvor gegenüber Mitpatienten in einer psychiatrischen Einrichtung angekündigt.

\section{Suizidmotiv}

Durch den Inhalt der aufgefundenen Abschiedsbriefe und die polizeilichen Ermittlungen konnten die in • Abb. 1 aufgeführten Motive für die gemeinsame Suizidabsicht eruiert werden. Bezüglich der Krankheiten ist anzumerken, dass zumeist ein Malignom in fortgeschrittenem Stadium vorlag.

\section{Suizidmethode}

In 20 Fällen wählten die Suizidenten jeweils die gleiche $(87,0 \%)$, in 3 Fällen jeweils unterschiedliche (13,0\%) Suizidmethoden.

In 10 Fällen $(43,5 \%)$ wurde eine substanzbedingte Intoxikation als weiche Suizidmethode gewählt, wobei diese lediglich in 4 Fällen für beide Beteiligte tödlich verlief; in 6 Fällen überlebte je- weils eine der Personen. In 4 Fällen fand ein Schlafmittel (2-mal Zolpidem, 2-mal Barbiturat), in 2 Fällen ein Benzodiazepin und in einem Fall eine Kombination aus beiden Anwendung. In einem weiteren Fall spritzten sich die Suizidenten Insulin; in einem anderen Fall nahmen sie Kaliumcyanid ein. Ein Suizidentenpaar, das sich bei einem stationären Entzug kennengelernt hatte, starb an einer Betäubungsmittelmischintoxikation.

In 7 Fällen (30,4\%) erfolgte ein Einsatz von Schusswaffen. Drei Frauen starben durch 2 Kopfschüsse, 3 Frauen und 6 Männer durch einen Kopfschuss. Der Mann schoss jeweils zuerst auf bzw. erschoss seine Ehefrau und anschließend sich selbst. Eine Ausnahme bildete ein Fall, bei dem der Mann nach der Schussabgabe auf seine Ehefrau, ohne eine Eigenschussabgabe zu vollführen, das gemeinsame Schlafzimmer in Brand setzte und an einer Kohlenmonoxidintoxikation verstarb. In einem Doppelsuizidfall wurde ebenfalls die Hauskatze erschossen.

Ein Ehepaar tötete sich durch Verbluten nach außen infolge scharfer Gewalteinwirkungen gegen die Unterarmbeugeseiten. Dies war ebenfalls die Todesursache bei einem Mann, der zuvor seine Ehefrau mit einem Bademantelgürtel erdrosselt hatte. Eine weitere Frau wurde durch ihren Ehemann erdrosselt, bevor dieser sich erhängte. In einem Fall wurde von den beiden männlichen Suizidenten ein Überrollen durch einen Zug gewählt.

In 2 Fällen wurden verschiedene Suizidmethoden kombiniert: In einem Fall leitete das Ehepaar über einen Schlauch Abgase in ein Auto; zusätzlich wurde ein Brand gelegt. In dem anderen Fall wählten die beiden Suizidenten eine „ungewöhnlich exzessive Ausgestaltung des Freitodes" [33]. Sie fuhren mit hoher Geschwindigkeit in einem Auto gegen einen Baum, hatten am Rücksitz befestigte Seile um die Hälse geschlungen und die Teppichbeläge mit Benzin durchtränkt. Zudem wiesen beide Suizidenten hochtoxische Werte für Doxepin sowie toxische Konzentrationen von Codein, Dihydrocodein und Paracetamol auf [33].

Die gewählten Suizidmethoden sind in - Abb. 2 aufgeführt. 
Rechtsmedizin 2021 · 31:42-49 https://doi.org/10.1007/s00194-020-00434-9

(c) Der/die Autor(en) 2020

F. Holz · M. A. Verhoff · J. Deschins · G. Duttge · M. Parzeller

\section{Der Doppelsuizid - Teil 2: rechtsmedizinische und kriminalistische Aspekte}

\section{Zusammenfassung}

Einleitung. Begehen zwei Menschen infolge einer gemeinsamen Entscheidung Suizid, wird dies als Doppelsuizid oder gemeinschaftlicher Suizid definiert. Die rechtsmedizinische Begutachtung hängt von den Umständen des konkreten Einzelfalls ab und kann für die rechtliche Würdigung insbesondere bei Konstellationen, bei denen ein Beteiligter überlebt hat, wichtige Hinweise liefern. Material und Methoden. Für einen 25-JahresZeitraum von 1995 bis 2019 wurden retrospektiv alle Sektionsfälle mit vollendeten und versuchten Doppelsuiziden analysiert und, soweit erhältlich, mit den Ermittlungsakten abgeglichen.

Ergebnisse. Unter den erhobenen 23 Doppelsuizidfällen fanden sich 16 vollendete sowie 7 Fälle mit jeweils einem Überlebenden. In $83 \%$ der Fälle handelte es sich um Ehepaare bzw. eingetragene Lebensgemeinschaften, in $13 \%$ um Personen, die sich zuvor in einer psychiatrischen Einrichtung, und in $4 \%$ um Personen, die sich in einem „Suizidforum “ im Internet kennengelernt hatten. Der Mittelwert des Sterbealters betrug bei den Männern 57,8 und bei den Frauen 63,4 Jahre. In etwa zwei Drittel der Fälle wurden Erkrankungen als vorherrschendes Suizidmotiv angegeben. Als häufigste Methode wurde eine Intoxikation gewählt. Am zweithäufigsten fanden Schusswaffen Anwendung, wobei ausnahmslos der Mann zuerst einen oder mehrere Kopfschüsse auf seine Frau abgab, bevor er sich suizidierte. Der häufigste Sterbe- und Auffindeort war das gemeinsame Bett der Suizidenten.

Diskussion. Im Gegensatz zu Suiziden i. Allg. wurden die Doppelsuizide am häufigsten mittels einer Intoxikation als sogenannte weiche Methode verübt. In Anlehnung an die rechtsmedizinische Literatur werden für einen Doppelsuizid typische Merkmale herausgearbeitet.

Schlussfolgerung. Doppelsuizide stellen seltene Fälle in der rechtsmedizinischen Praxis dar, wobei die Abgrenzung zu einem erweiterten Suizid (Homizid-Suizid) schwierig werden kann und zugleich unabdingbar ist. Hierfür ist eine ganzheitliche Berücksichtigung der Vorgeschichte, der rechtsmedizinisch erhobenen Befunde und der kriminalistischen Umstände erforderlich.

\section{Schlüsselwörter}

Gemeinschaftliche Selbsttötung · Selbstmordpakt · Erweiterter Suizid · Homizid-Suizid · Philemon und Baucis

\section{The double suicide-Part 2: forensic and criminalistic aspects}

\section{Abstract}

Introduction. When two people commit suicide as a result of a mutual decision, this is defined as a double suicide or a suicide pact. Forensic medical evaluation should include the circumstances of the individual case and can provide important information for the legal assessment, especially in cases when one person survived.

Material and methods. For the 25-year period from 1995 to 2019 , autopsy cases of completed and attempted double suicides were retrospectively analyzed including the investigation files, if they were available. Results. The study revealed 23 double suicides among which 16 suicides were completed and in 7 cases one partner survived. Married couples or persons living in registered partnerships were involved in $83 \%$ of these cases and in 13\% the people concerned had previously met in a psychiatric institution and $4 \%$ had met in an online suicide chat room. The mean age at the time of death was 57.8 years for males and 63.4 years for females. In approximately two thirds of the cases diseases were reported as the main suicidal motive. The most frequent method of suicide was intoxication, with the use of firearms being the second most common method. Without exception, the man first shot his wife with one or more shots to the head before killing himself. In most cases the shared bed was the place where the deceased were found.
Discussion. In contrast to suicides in general, double suicides were most often committed by intoxication as a so-called soft method. Based on the forensic literature, typical features of a double suicide are discussed. Conclusion. In forensic medicine, double suicides are rare; however, the distinction from an extended suicide (homicide-suicide) is essential but can be difficult to achieve. The case history, forensic findings and criminal investigations of the circumstances have to be considered.

\section{Keywords}

Double suicide · Suicide pact $\cdot$ Extended suicide $\cdot$ Homicide-suicide $\cdot$ Philemon and Baucis

\section{Toxikologische Untersuchungen}

Zusätzlich zu den 10 Fällen, bei denen eine Intoxikation als Suizidmethode gewählt wurde, ordneten die Ermittlungsbehörden in 5 Fällen die Untersuchungen auf Alkohol, Medikamente und Drogen sowie bei 3 Suizidenten auf Kohlenmonoxid an. Die Blutalkoholkonzentration der Suizidenten lag im Median bei 0,17\%o (Mittelwert $=0,34 \%$, Min. $=0,00 \%$; Max. $=1,66 \%$ o). Abgesehen von den to- desursächlichen Intoxikationen konnte in 2 Fällen die Aufnahme körperfremder Substanzen nachgewiesen werden (einmal Benzodiazepine, einmal Mischintoxikation Morphin, Codein, THC, Doxepin).

\section{Ort des Doppelsuizids}

In 18 Doppelsuizidfällen (78,3\%) wählten die Suizidenten ihr eigenes zu Hause als Sterbeort: Zehn Doppelsuizide
$(43,5 \%)$ wurden im gemeinsamen Bett vollzogen, $5(21,7 \%)$ gemeinsam im Wohnzimmer bzw. in der Küche bzw. in der Garage. In 3 Fällen (13,0 \%) wurden die Leichen an verschiedenen Orten innerhalb des Hauses bzw. der Wohnung aufgefunden.

Die restlichen 5 Fälle (21,7\%) ereigneten sich in einem Hotelzimmer bzw. in der Öffentlichkeit - auf einer Bundesstraße, in einem Gleisbett, auf einer Waldlichtung und vor einer Kapelle. 


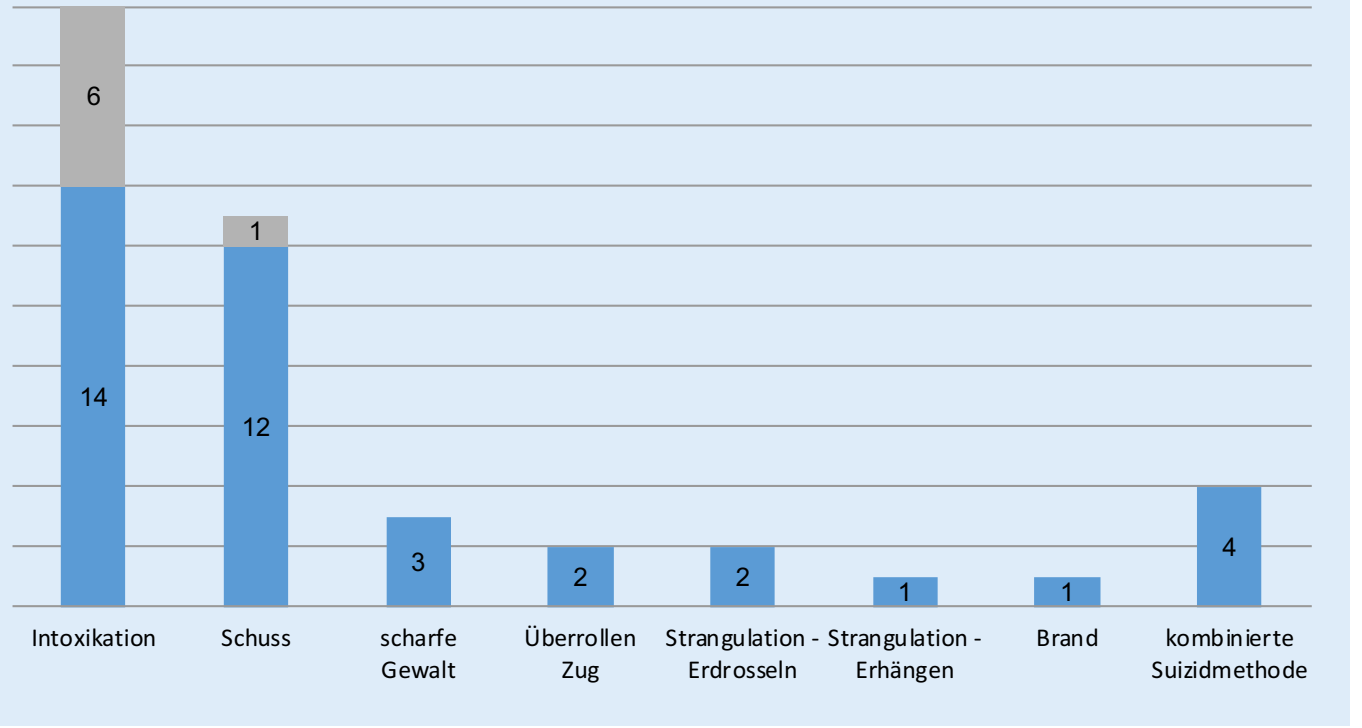

Abb. $2 \triangleleft$ Anzahl der gewählten Suizidmethoden - vollendeter Suizid blau, fehlgeschlagener Suizid grau

\section{Einseitig überlebter Doppelsuizid}

In 7 Fällen überlebte jeweils ein Beteiligter den Doppelsuizid, es handelte sich um 3 Männer und 4 Frauen. Wie in - Abb. 2 aufgeführt, wurde in 6 dieser Fälle eine Intoxikation als Suizidmethode gewählt. Überlebt wurden 2 Suizidversuche mit Zolpidem sowie jeweils einer mit einem Benzodiazepin, einem Barbiturat und einer Kombination aus Benzodiazepin und Zolpidem sowie eine Insulinüberdosierung. Eine Frau überlebte einen Kopfschuss, der schussausführende Mann hingegen nicht.

\section{Diskussion}

In der Bundesrepublik Deutschland werden aktuell jährlich ca. 10.000 Suizidtodesfälle erfasst [50]. Somit versterben deutlich mehr Menschen infolge eines Suizides als beispielsweise infolge von Verkehrsunfällen und Betäubungsmittelintoxikationen zusammen [50-52]. Bemerkenswert hierbei ist, dass der Anteil von Suiziden an allen Todesursachen mit zunehmendem Lebensalter sinkt, während die Suizidrate deutlich ansteigt (2017 jeweils pro 100.000 Einwohner, z. B.: 20 bis 24 Jahre $n=6,6$; 40 bis 44 Jahre $n=9,3 ; 60$ bis 64 Jahre $n=14,0 ; 80$ bis 84 Jahre $n=28,5$ und für 90 Jahre und älter $n=30,5$ [51]) und über 70Jährige weltweit die höchste Suizidrate aufweisen [44]. In der Literatur wird der Anstieg der Suizidrate mit zunehmendem Lebensalter als „Ungarisches Muster" bezeichnet [46, 47, 55].

\section{Geschlecht, Alter, Beziehung}

In der vorliegenden Studie stellten die Doppelsuizidenten einen Anteil von $0,24 \%$ an allen rechtsmedizinischen Sektionsfällen dar. Bezogen auf alle obduzierten Suizidfälle entspricht dies ca. $1,8 \%$, was vergleichbar ist mit den in der Literatur angegebenen Häufigkeiten von $0,0007-4,0 \%$ an allen Suiziden $[3,5$, $10,11,17,18,23,26,27,34,36,42,43$ ], wobei Japan die höchsten Doppelsuizidraten aufweist $[14,17,34]$. Dabei ist jedoch zu berücksichtigen, dass häufig vermeintliche „eindeutige“ Einzelsuizide keiner gerichtlichen Leichenöffnung durch die Ermittlungsbehörden zugeführt werden, während Doppelsuizide eher obduziert werden, sodass ein entsprechendes Dunkelfeld im Vergleich zu Einzelsuiziden geringer sein dürfte. Für Deutschland wird eine jährliche Fallzahl an Doppelsuiziden von 50-100 vermutet [11].

Im Gegensatz zum Einzelsuizid, mit einem seit mehreren Jahren annähernd konstanten Geschlechterverhältnis von 3 $\left(\sigma^{7}\right): 1$ (ㅇ) [7, 51, 55], zeigten zahlreiche englisch- und deutschsprachige Studien mit einer Fallzahl von mehr als 5 vollendeten Doppelsuiziden eine deutliche Verschiebung zum weiblichen Ge- schlecht - die erhobenen Relationen rangierten zwischen 1 ( $\left.\sigma^{7}\right): 1$ (ㅇ) (6 Fälle [43]) über 1,3 $\left(\sigma^{7}\right): 1$ (ㅇ) (278 Fälle [28]) bis $1,6\left(\sigma^{7}\right): 1$ (우) (7 Fälle [30]). Die in der vorliegenden Studie erhobene Geschlechterverteilung von 1,2 ( $\left.\sigma^{7}\right): 1$ (ㅇ) stützt diese Beobachtungen. Hintergrund dieser Verschiebung sind zumeist aus einer männlichen und einer weiblichen Person bestehende Doppelsuizidpaare [27, 30, 34, 43]. Nur gelegentlich finden sich in der westlichen Literatur beschriebene Fälle von gleichgeschlechtlichen (befreundeten) Doppelsuizidpaaren [2,33] oder, wie auch in einem Fall dieser Studie, homosexuellen Paaren [21, 28, 30]. Entgegen anderer Publikationen waren im vorliegenden Untersuchungsgut keine Doppelsuizide von Geschwistern [26,30,40] bzw. Zwillingen [16] vertreten.

Während auf Suizid spezialisierte Foren im Internet bereits seit Längerem genutzt werden, um einen „Suizidpartner" zu finden, rücken in jüngerer Zeit vermehrt Social-Media-Plattformen (z. B. Facebook, Instagram, Twitter) in den Vordergrund. Lee und Kwon publizierten, dass innerhalb von 43 Tagen 551 verschiedene Twitter-Nutzer 1702 Kurznachrichten im Hinblick auf einen gemeinsamen Suizid posteten [35]. In der vorliegenden Studie lernte sich ein Doppelsuizidpaar auf einem Suizidforum kennen. Das teilweise beschriebene, dem Doppelsuizid ähnliche Phänomen des Mehrfachsuizids durch mehr als 2 


\section{Originalien}

Tab. 2 Relevante Kriterien beim Doppelsuizid

Beziehungsebene

1) Enge, langjährige Partnerbeziehung zwischen den Beteiligten in einem höheren bis zumeist hohen Lebensalter

2) Jüngere Beteiligte, meist junge Erwachsene, mit kurzer, auf einem gemeinsamen Aufenthalt in einer psychiatrischen Einrichtung gründenden Beziehung

Doppelsuizidmotive

Zu 1) Schwere körperliche Erkrankung eines oder beider Beteiligten als vorherrschendes Suizidmotiv, häufig soziale Isolation ("Isolation zu zweit" [11]), ggf. psychische Erkrankung bei einem oder beiden Ehepartner(n)

Zu 2) Psychische Erkrankungen und/oder Betäubungsmittelabhängigkeit der Beteiligten

Ankündigung bzw.

Motiverläuterung

Kurzfristige vorherige mündliche Ankündigung und/oder Auffinden eines von beiden Suizidenten unterschriebenen Abschiedsbriefes und/oder Testaments

Zeitliche Abfolge der (Selbst-)Tötungs-

handlungen

Suizidmethode

Überwiegend Anwendung der gleichen Methode

Ort des Doppelsuizids

Vornehmlich in engem räumlichem Zusammenhang im eigenen Haus, insbesondere im gemeinsamen Bett

Alter

Volljährigkeit

Sozioökonomischer

Status

Meist höherer sozioökonomischer Status der Beteiligten
Ehepaares und den Liebespaarsuizid, der wohl in Anlehnung an „Romeo und Julia“ zu verstehen ist [19]. Während Ersterer in über $80 \%$ der vorliegenden Studie vorlag, konnte kein Fall ausfindig gemacht werden, der eindeutig eines der von Grellner und Krull angeführten Kriterien eines „konventionellen Liebespaarsuizids" (Missbilligung der Umgebung, Unmöglichkeit des Zusammenkommens, Wunsch der Vereinigung im Tod) aufwies [19]. Liebespaarsuizide („love suicide“: „shinjū“; eine Jahrhunderte alte Tradition, Heine [25]) wurden v. a. aus Japan berichtet (bis $\mathrm{zu} 74,5 \%$ [14]). Kulturelle und soziale Unterschiede werden daher in der Literatur aufgezeigt und für westliche Kulturen v. a. auf das Phänomen des Doppelsuizids bei älteren, erkrankten Paaren, teils mit Depressionen hingewiesen [7, 22].

Als weiterer Aspekt des Doppelsuizids konnten in unserer Studie 3 Fälle aufgezeigt werden, bei denen sich die jeweiligen Suizidenten zuvor in einer psychiatrischen Einrichtung kennengelernt hatten. Bei einem weiteren Doppelsuizidfall lernten sich die Beteiligten im Internet kennen; einer der Beteiligten war wenige Tage vor dem Suizid aus einer psychiatrischen Klinik entlassen worden. Diese Konstellationen verdienen im Hinblick auf eine mögliche Prävention gesonderte Erwähnung, da sich die ersten Monate nach der Entlassung als durchaus vulnerable Phase für eine Suizidbegehung herausstellen können [47]. Nach Hunt et al. [28] begingen etwa $30 \%$ der Patienten innerhalb der ersten 3 Monate nach der Entlassung aus der Psychiatrie einen Suizid. Sie bevorzugten härtere Suizidmethoden, was sich mit den vorliegenden Ergebnissen deckt. Im Gegensatz zu den restlichen Fällen von Doppelsuiziden waren bei diesen 4 Fällen ausschließlich Suizidenten in einem Alter zwischen 18 und 24 Jahren betroffen (Mittelwert 20,7 Jahre; Median 21 Jahre). Dieser Umstand und die Tatsache, dass in unserer Studie alle beteiligten Personen zuvor in der psychiatrischen Einrichtung Suizidäußerungen getätigt hatten bzw. laut Hunt et al. 36\% der Beteiligten ihre letzte psychiatrische Konsultation vor dem Doppelsuizid nicht mehr wahrgenommen hatten, ergibt ein weiteres Kollektiv von möglichen Dop- 
pelsuizidenten. Hierzu finden sich in der Literatur neben Hunt et al. [28] bereits 2 Fallberichte [33, 38].

Vorherrschendes Motiv in unserem Untersuchungskollektiv war, in Übereinstimmung mit der westlichen Literatur, eine schwere körperliche Erkrankung (meist Malignom) eines oder beider Suizidenten ([13, 41, 43]; prominente Beispiele bei Csef [11]). Ferner gilt Einsamkeit als zentraler Faktor für Doppelsuizide [55, 57]. In der Literatur finden sich Beschreibungen wie „Isolation zu zweit" [11], „encapsulated unit“ [26], „too lonely to die alone“ [39] oder „Zwei-Einigkeit“, die durch „Starrheit und Inflexibilität“ mit der Unfähigkeit $\mathrm{zu}$ weitergehenden Sozialkontakten [22] gekennzeichnet ist.

Hinsichtlich des Vorliegens einer depressiven Erkrankung als Prädisposition für das Begehen eines Doppelsuizids widerspricht sich die Literatur: Während beispielsweise Autoren wie Fishbrain [15] und Hemphill [26] angeben, dass Depressionen bei Doppelsuizidenten „nicht häufig“ bzw. im Gegensatz zu Einzelsuiziden weniger häufig vorliegen, vertreten andere Autoren die Meinung, dass meist zumindest einer der beiden Beteiligten an einer Depression erkrankt war bzw. ist und z.T. bereits vor dem Doppelsuizid Suizidversuche unternommen hat $[6,34,45]$. Demgegenüber werden vornehmlich aus ostasiatischen Ländern vermehrt bzw. teils auch vorrangig die Unmöglichkeit des Auslebens einer Liebe als Motiv für einen Doppelsuizid genannt $[1,14,19,37]$.

\section{Ort des Doppelsuizids}

Die Auffindesituation von etwa $80 \%$ der Fälle im eigenen Haus sowie die unmittelbare Nähe der Verstorbenen zueinander decken sich weitgehend mit den Angaben in der Literatur von beispielsweise $83 \%$ bei Prat et al. [43] bzw. bei Pollak als „Tatbegehung an derselben Örtlichkeit (meist Wohnbereich)“ [41] oder generell bei älteren Suizidenten [8]. Die Ausführung des Doppelsuizids im gemeinsamen Ehebett unterstreicht zudem die enge Verbundenheit der Partner.

\section{Suizidmethode}

Im Allgemeinen werden Einzelsuizidversuche im Alter mit einer höheren Letalintensität durchgeführt, wobei der Literatur zufolge in den Altersgruppen ab 60 Jahren die Anwendung sog. harter Suizidmethoden überwiegt $[47,57]$. Bei beiden Geschlechtern wurde sowohl von Schmidtke et al. als auch von Wulff et al. bei Einzelsuiziden das Erhängen als häufigste Methode beobachtet [47, 57]; bei Männern findet sich als zweithäufigste Methode der Schusswaffengebrauch, bei Frauen die Intoxikationen [47].

Die in dieser Studie untersuchten Suizide verliefen in $84,9 \%$ tödlich, was sowohl die in der Literatur beschriebene hohe Letalintensität [47, 57] als auch die von Hunt et al. beschriebene Rate an fehlgeschlagenen Doppelsuizidversuchen von $16 \%$ stützt [28]. Differenziert nach Doppelsuizidpaaren verstarben ausschließlich in 73,9\% jeweils beide Beteiligten. Diese Zahl verdeutlicht die juristische Relevanz verschiedener Konstellationen nach fehlgeschlagenem Suizid eines Beteiligten [12].

Hinsichtlich der angewandten Suizidmethoden waren gegenüber Einzelsuiziden in der vorliegenden Studie deutliche Unterschiede erkennbar. Wie bereits von Le Bihan und Bénézech beschrieben [34], fand sich auch im Frankfurter Kollektiv geschlechtsunabhängig als häufigste die „weiche“ Methode der Intoxikation, die von ca. einem Drittel der Suizidenten überlebt wurde. Als zweithäufigste Methode wurde der Schuss gewählt. Hierbei war sowohl auffällig, dass ausschließlich Kopfschüsse erfolgten, und dass in allen Fällen der Mann zuerst auf seine Ehefrau schoss bzw. sie erschoss und anschließend sich suizidierte. Diese Konstellation wurde bereits von Faller-Marquardt und Pollak beschrieben [13]. Binder et al. publizierten einen Doppelsuizidfall, bei dem sich die Beteiligten eng umschlungen mit einer Schussabgabe suizidierten [4]. Die von Fishbrain et al. bei den von ihnen untersuchten 20 Doppelsuizidfällen getätigte Beobachtung, dass vornehmlich „nonviolente“ Suizidmethoden gewählt wurden ([14], siehe auch [7]), konnte anhand dieser Studie somit nicht bestätigt werden. Die Verwendung harter Suizid- methoden zur Durchführung des Doppelsuizids wird ebenfalls in einer Studie aus Serbien vermerkt, wo Handgranaten bei 6 Doppelsuizidfällen zum Einsatz kamen und in 5 Fällen diese von der Frau gezündet wurden [40].

Der von uns festgestellte Prozentsatz (87\%) lag unter dem von Prat et al. publizierten Prozentsatz (100\%), wobei Prat et al. ausschließlich 6 Doppelsuzidfälle ausgewertet hatten [43]. In 2 Fällen dieser Studie $(8,7 \%)$ wurde eine kombinierte Suizidmethode gewählt. Ein solcher Doppelsuizid, den wir als suizidalen Overkill bezeichnen, wird äußerst selten in der Literatur beschrieben [29, 33].

Zusammenfassend konnten in Anlehnung an Pollak [41], Prat et al. [43] und Große Perdekamp et al. [20] unter Einbeziehung der Sektionsfälle des Instituts für Rechtsmedizin Frankfurt am Main von 1995-2019, wenngleich auf geringer Fallzahl, die in • Tab. 2 aufgeführten Merkmale als typisch für den Doppelsuizid verifiziert und teils erweitert werden. In Ermangelung näherer Informationen, z.B. aus den Ermittlungsakten, konnte der durch Prat et al. sowie Brown und Barraclough beschriebene hohe sozioökonomische Status der beteiligten Personen ebenso wenig wie der von Brown und Barraclough beschriebene Umstand der Kinderlosigkeit der Doppelsuizidenten $(77 \%)$ untersucht werden $[5,43]$.

\section{Fazit für die Praxis}

Das Phänomen des Doppelsuizids ist lange bekannt, und die Todesumstände von Persönlichkeiten wie Heinrich von Kleist und Henriette Vogel (mit unterschiedlicher Bewertung in der Literatur) werden in diesem Zusammenhang genannt. Wenngleich es sich um ein seltenes Ereignis handelt, ist die Notwendigkeit der Abgrenzung zu einem erweiterten Suizid unabdingbar. Hierfür können Merkmale wie beispielsweise ein hohes Lebensalter, schwere Erkrankung einer oder beider Beteiligten und das Fehlen von Abwehrspuren, aber insbesondere Dokumente zur Feststellung der Motivlage Hinweise geben. In dieser Studie wurde als weiteres Kollektiv von Doppelsuizidenten jüngere Erwachsene mit kurzer, auf einem gemeinsamen Aufent- 
halt in einer psychiatrischen Einrichtung gründender Beziehung festgestellt. Dieser Aspekt ist aus ärztlicher Sicht nicht zu vernachlässigen, da sich hieraus präventive Ansätze für psychiatrisch tätige Mediziner ergeben können. Neben einer umfassenden rechtsmedizinischen Begutachtung, einschließlich toxikologischen Untersuchungen, sollte sich die Unterscheidung zwischen Doppelsuizid und Homizid-Suizid auf eine ganzheitliche Berücksichtigung der Vorgeschichte und der kriminologischen Umstände gründen. Insbesondere bei einem einseitig fehlgeschlagenen Doppelsuizid können eine penible Tatrekonstruktion sowie die Klärung der Motivlage vor der Tatbegehung als auch das Nachtatverhalten von enormer Relevanz für eine spätere juristische Aufarbeitung des Geschehens sein. Nach Doppelsuiziden ist zudem ein Augenmerk auf die trauernden Angehörigen zu richten, und diesen sind entsprechende Hilfsangebote nahezulegen.

„Ein altes, kluges Paar hat sich umgebracht und nicht demütig gewartet, bis der Tod einen nach dem anderen holt und sie also scheidet."

(Aus dem Nachruf für den Philosophen André Gorz und seine Ehefrau Dorine [54])

\section{Korrespondenzadresse}

\section{Dr. med. F. Holz}

Institut für Rechtsmedizin des Universitätsklinikums, Goethe-Universität Frankfurt am Main

Kennedyallee 104, 60596 Frankfurt am Main, Deutschland

holz@med.uni-frankfurt.de

Danksagung. Die Autoren danken Herrn Prof. Dr. Mebs für die Übersetzung der Zusammenfassung ins Englische.

Funding. Open Access funding enabled and organized by Projekt DEAL.

\section{Einhaltung ethischer Richtlinien}

Interessenkonflikt. F. Holz, M.A. Verhoff, J. Deschins, G. Duttge und M. Parzeller geben an, dass kein Interessenkonflikt besteht.
Ein Votum der Ethikkommission und ein Datenschutzkonzept lagen vor

Open Access. Dieser Artikel wird unter der Creative Commons Namensnennung 4.0 International Lizenz veröffentlicht, welche die Nutzung, Vervielfältigung, Bearbeitung, Verbreitung und Wiedergabe in jeglichem Medium und Format erlaubt, sofern Sie den/die ursprünglichen Autor(en) und die Quelle ordnungsgemäß nennen, einen Link zur Creative Commons Lizenz beifügen und angeben, ob Änderungen vorgenommen wurden.

Die in diesem Artikel enthaltenen Bilder und sonstiges Drittmaterial unterliegen ebenfalls der genannten Creative Commons Lizenz, sofern sich aus der Abbildungslegende nichts anderes ergibt. Sofern das betreffende Material nicht unter der genannten Creative Commons Lizenz steht und die betreffende Handlung nicht nach gesetzlichen Vorschriften erlaubt ist, ist für die oben aufgeführten Weiterverwendungen des Materials die Einwilligung des jeweiligen Rechteinhabers einzuholen.

Weitere Details zur Lizenz entnehmen Sie bitte der Lizenzinformation auf http://creativecommons.org/ licenses/by/4.0/deed.de.

\section{Literatur}

1. Avis S, Hutton C (1994) Dyadic suicide-a case study. Am J Forensic Med Pathol 15:18-20

2. Balázs J, Keresztény A, Pelbát G, Sinka L, Szilvás F, Torzsa T(2013) Online media reporton a Hungarian double suicide case: comparison of consecutively published articles. Psychiat Danub 25:248-254

3. Behera C, Rautji R, Kumar R, Pooniya S, Sharma P, Gupta SK (2017) Double hanging with single ligature: an unusual method in suicide pact. JForensic Sci 62:265-266

4. Binder S, Banaschak S, Beike J, Rothschild MA, Kamphausen T (2018) Vom Rücken durch die Brust ins Auge - Ungewöhnlicher gemeinschaftlicher Suizid mittels einer Schusswaffe. Rechtsmedizin 28:423-428

5. Brown M, Barraclough B (1997) Epidemiology of suicide pacts in England and Wales, 1988-92. BMJ 315:286-287

6. Brown M, Barraclough B (1999) Partners in life and in death: the suicide pact in England and Wales 1988-1992. Psychol Med 29:1299-1306

7. Byard RW (2016) The features and complexities of coincident deaths. Forensic Sci Med Pathol 12:1-3

8. Cattell HR (1988) Elderly suicide in London: an analysis of coroners' inquests. Int J Geriatr Psychiatry 3:251-261

9. Ciesiolka S, Riße M, Busch B, Verhoff MA (2008) Philemon and Baucis Death? Two cases of double deaths of married couples. Forensic Sci Int 176:e7-e10

10. Cohen J (1961) A study of suicide pacts. Med Leg J 29:144-151

11. Csef H (2016) Doppelsuizide von Paaren nach langer Ehe. Verzweiflungstaten oder Selbstbestimmung bei unheilbaren Krankheiten? Int Z Philos Psychosom 8(1):1-10

12. Deschins J, HolzF, Duttge G, VerhoffMA, ParzellerM (2020) Der Doppelsuizid - Teil 1: Juristische Aspekte und historische/zeitgeschichtliche Fälle. Rechtsmedizin. https://doi.org/10.1007/s00194020-00433-w
13. Faller-Marquardt M, Pollak S (2000) Gemeinschaftlicher Suizid mit Schussabgabe in die Scheitelregion. Rechtsmedizin 10:148-152

14. Fishbrain DA, Aldrich TE (1985) Suicide pacts: international comparison. J Clin Psychiatry 46:11-15

15. Fishbrain DA, d'Achille L, Barsky S, Aldrich TE (1984) A controlled study of suicide pacts. J Clin Psychiatry 63:41-44

16. Giusti GV, Chiarotti M (1981) Double 'suicide' by chloroform in a pair of twins. Med Sci Law 21:2-3

17. Granboulan V, Zivi A, Basquin M (1997) Double suicide attempt among adolescents. J Adolesc Health 21:128-130

18. Gregory M (2013) Dying together: suicide pacts and other episodes of paired suicides in Yorkshire and the Humber. Br JSoc Work 43:298-316

19. Grellner W, Krull F (1996) Ungewöhnlich motivierter Doppelsuizid eines lesbischen Liebespaares - Phänomenologie, Psychodynamik und Zeitgeisteinflüsse. Arch Kriminol 198:65-72

20. Große Perdekamp M, Pollak S, Thierauf A (2010) Medicolegal evaluation of suicidal deaths exemplified by the situation in Germany. Forensic Sci Med Pathol 6:58-70

21. Janßen K, RothschildMA, Kamphausen T(2018)Die verpackte Leiche. Auswertung des Sektionsgutes von 2009-2016 hinsichtlich unterschiedlicher Fallumstände und Motivationen. Rechtsmedizin 28:1-9

22. Haenel T (2001) Suizid und Zweierbeziehung. Vandenhoeck \& Ruprecht, Göttingen

23. Haenel T, Elsässer PN (2000) Double suicide and homicide-suicide in Switzerland. Crisis 21:122-125

24. HaenelT(1996) Der Suizid als Gruppen-und Kollektivphänomen. In:Wenglein E, Hellwig A, Schoof M (Hrsg) Selbstvernichtung. Psychodynamik und Psychotherapie bei autodestruktivem Verhalten. Vandenhoeck \& Ruprecht, Göttingen/Zürich, S 75-88

25. Heine S (1994) Tragedy and salivation in the floating world: Chikamatsu's double suicide drama as Millenarian discourse. J Asian Stud 53:367-393

26. Hemphill RE, Thornley FI (1969) Suicide pacts. S Afr Med J43(44):1335-1338

27. Hocaoglu C (2009) Double suicide attempt. Singapore Med J 50:e81-e84

28. Hunt I, While D, Windfuhr K, Swinson N, Shaw J, Appleby L, Kapur N (2009) Suicide pacts in the mentally ill: a national clinical survey. Psychiatry Res 167:131-138

29. Jayanth SH, Chandra G, Hugar BS, Kainoor S (2014) Secondary combined suicide pact. J Forensic Leg Med 23:76-79

30. Jensen LL, Gilbert JD, Byard RW (2009) Coincident deaths: double suicide or murder-suicide? Med Sci Law 49:27-32

31. Jübner M, Hatz D, Lyness J, Röhrich J, Riepert T, Rothschild MA (2011) Zwei Fälle von Dreifachsuiziden mithilfe von Holzkohle - Auffällige Duplizität zweier Mehrfachsuizide. Rechtsmedizin 21:111-115

32. Lardi C, Schmit G, Burkhardt S, Mangin P, Palmiere C (2014) Philemon and Baucis deaths: case reports and postmortem biochemistry contribution. JForensic Sci 59:1133-1138

33. Lasczkowski G, Röhrich J, Brazke H (1998) Suizidexzeß - Darstellung eines ungewöhnlichen Falles. Arch Kriminol 202:100-108

34. Le Bihan P, Bénézech $M$ (2006) Pactes suicidaires: une revue de la literature. Ann Med Psychol 164:292-303 
35. Lee SY, Kwon Y (2018) Twitter as a place where people meet to make suicide pacts. Public Health 159:21-26

36. Lester D (1997) The sexual politics of double suicide. Fem Psychol 7:148-154

37. Milin R, Turgay A (1990) Adolescent couple suicide: literature review. Can J Psychiatry 35(2):11-15

38. Moutian W, Schmitt G, Mattern R (1994) Suizid mit Prothipendyl. Arch Kriminol 193:158-162

39. Ozawa-de Silva C (2008) Too lonely to die alone: Internet suicide pacts and existential suffering in Japan. Cult Med Psychiatry 34:516-551

40. Petković S, Maletin M, Drašković D (2013) Nonterrorist suicides using hand grenades on the territory of the Autonomous Province of Vojvodina, Serbia, from 1990 to 2009. Int J Legal Med 127:225-229

41. Pollak S (2005) Rechtsmedizinische Aspekte des Suizids. Rechtsmedizin 15:235-249

42. Pollak S (1978) Gemeinschaftliche Selbstmorde. Beitr Gerichtl Med 36:53-59

43. Prat S, Rérolle C, Saint-Martin P (2013) Suicide pacts: six cases and literature review. J Forensic Sci 58(4):1092-1098

44. Richards N (2017) Old age rational suicide. Sociol Compass 11:e12456

45. Rosenbaum M(1983)Crimeand punishment-the suicide pact. Arch Gen Psychiatry 40:979-982

46. Rübenach SP (2007) Todesursache Suizid. Wirtschaft und Statistik. DeStatis, Wiesbaden, S960-971

47. Schmidtke A, Sell R, Löhr C (2008) Epidemiologie von Suizidalität im Alter. Z Gerontol Geriat 41:3-13

48. Schwab G (2001) Sagen des klassischen Altertums. Ueberreuter, Wien, S101-103

49. Siems A, Flaig B, Ackermann H, Verhoff MA, Parzeller M (2017) Homicide-suicide-postmortem study from the Institute of Legal Medicine in Frankfurt/Main from 1994 to 2014. Rechtsmedizin 27:175-184

50. Statista (2020) Anzahl der Sterbefälle durch vorsätzliche Selbstbeschädigung (Suizide) in Deutschland in den Jahren von 1980 bis 2018 https://de.statista.com/statistik/daten/studie/ 583/umfrage/sterbefaelle-durch-vorsaetzlicheselbstbeschaedigung/.Zugegriffen: 10. Mai 2020

51. Statista (2020) Selbstmordrate in Deutschland nach Altersgruppe in den Jahren 2014 bis 2018. https://de.statista.com/statistik/daten/ studie/318224/umfrage/selbstmordrate-indeutschland-nach-altersgruppe/.Zugegriffen: 10 Mai 2020

52. Statistisches Bundesamt (2020) Suizide. https:// www.destatis.de/DE/Themen/GesellschaftUmwelt/Gesundheit/Todesursachen/Tabellen/ suizide.html.Zugegriffen: 26. Mai 2020

53. Stockhausen S, Wöllner K, Möhle F, Doberentz $E_{\text {, }}$ Kernbach-Wighton G, Madea B (2017) Erweiterter Suizid, Doppelsuizid oder Homizid mit nachfolgendem Suizid? 2 Falldarstellungen. Arch Kriminol 239:87-88

54. von Thadden E (2007) André Gorz: Über den Tod hinaus. Die Zeit von 28.09.2007. http://www.zeit. de/2007/40/Nachruf-Gorz. Zugegriffen: 7. Sept. 2020

55. Wolfersdorf M (2012) Suizid und Suizidalität aus psychiatrisch-psychotherapeutischer Sicht. Psychother Dialog 13:2-7

56. WolfersdorfM (2010) Der Doppelsuizid (-versuch). Charakteristika erweiterter suizidaler Handlungen. Suizidprophylaxe 37:92-101

57. Wulff $B$, Iwersen-Bergmann S, Pabel T, Püschel K (2014) Suizide von über 80-jährigen in Hamburg unter Berücksichtigung geschlechtsspezifischer Aspekte. Rechtsmedizin 24:103-106

\section{Alle Inhalte von springermedizin.de online im Volltext lesen}

Mit e.Med Interdisziplinär - dem Kombi-Abo von SpringerMedizin.de - können Sie jederzeit auf alle Inhalte zugreifen, die Sie für Ihren Praxis- oder Klinikalltag benötigen. Sie sind immer aktuell informiert - über die neuesten Publikationen, wichtige Studien oder innovative Therapieverfahren.

Die Vorteile von e.Med Interdisziplinär:

- Gestalten Sie Ihre Fortbildung nach Ihren Bedürfnissen:

e.Med Interdisziplinär bietet Ihnen Online-Zugang zu 600 CME-Fortbildungskursen aller Fachrichtungen

- Sie entscheiden, was Sie lesen möchten:

Alle Fachzeitschriften stehen digital im Volltext zur Verfügung.

- Finden Sie die gewünschten Informationen auch in englischen Publikationen: Sie können in mehr als 500 englischsprachigen Fachzeitschriften online recherchieren und auf die Suchergebnisse uneingeschränkt im Volltext zugreifen. - Auf Wunsch erhalten sie darüber hinaus eine gedruckte Fachzeitschrift nach Wahl.

NEU: Mit den fachspezifischen e.Med-Abos können Sie sich effizient innerhalb Ihres Fachgebietes auf dem Laufenden halten. Das Angebot reicht von AINS bis Radiologie - Sie haben die Wahl!

Testen Sie e.Med kostenlos und unverbindlich!

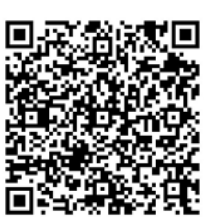

Jetzt informieren unter www.springermedizin.de $\Rightarrow$ „Abos“ oder telefonisch unter 0800-77 80777

(Montag bis Freitag, 10 bis 17 Uhr) 